\title{
Regulation and Restoration of Motoneuronal Synaptic Transmission During Neuromuscular Regeneration in the Pulmonate Snail Helisoma trivolvis
}

\author{
M. B. TURNER ${ }^{1}$, T. M. SZABO-MAAS ${ }^{2, \dagger}$ J. C. POYER ${ }^{3}$, AND M. J. ZORAN ${ }^{3, *}$ \\ ${ }^{1}$ Department of Cellular Biology, University of Georgia, Athens, Georgia 30602; ${ }^{2}$ Department of \\ Biology, Brandeis University, Waltham, Massachusetts 02453; and ${ }^{3}$ Department of Biology, Texas A\&M \\ University, College Station, Texas 77843
}

\begin{abstract}
Regeneration of motor systems involves reestablishment of central control networks, reinnervation of muscle targets by motoneurons, and reconnection of neuromodulatory circuits. Still, how these processes are integrated as motor function is restored during regeneration remains ill defined. Here, we examined the mechanisms underlying motoneuronal regeneration of neuromuscular synapses related to feeding movements in the pulmonate snail Helisoma trivolvis. Neurons B19 and B110, although activated during different phases of the feeding pattern, innervate similar sets of muscles. However, the percentage of muscle fibers innervated, the efficacy of excitatory junction potentials, and the strength of muscle contractions were different for each cell's specific connections. After peripheral nerve crush, a sequence of transient electrical and chemical connections formed centrally within the buccal ganglia. Neuromuscular synapse regeneration involved a three-phase process: the emergence of spontaneous synaptic transmission (P1), the acquisition of evoked potentials of weak efficacy (P2), and the establishment of functional reinnervation (P3). Differential synaptic efficacy at muscle contacts was recapitulated in cell culture. Differences in motoneuronal presynaptic properties (i.e., quantal content)
\end{abstract}

Received 12 April 2010; accepted 5 May 2010.

* To whom correspondence should be addressed. E-mail: zoran@mail. bio.tamu.edu

$\dagger$ Current address: Department of Biology, Delaware State University, Dover, Delaware 19904.

Abbreviations: Aj, anterior jugalis; AP-PSP, action potential-evoked postsynaptic potentials; DM, defined medium; EJP, excitatory junction potential; Lbn, laterobuccal nerve; mEJP, miniature excitatory junction potential; mPSP, miniature postsynaptic potential; PSP, postsynaptic potential; Slrt, supralateral radular tensor; Vbn, ventrobuccal nerve. were the basis of disparate neuromuscular synapse function, suggesting a role for retrograde target influences. We propose a homeostatic model of molluscan motor system regeneration. This model has three restoration events: (1) transient central synaptogenesis during axonal outgrowth, (2) intermotoneuronal inhibitory synaptogenesis during initial neuromuscular synapse formation, and (3) target-dependent regulation of neuromuscular junction formation.

\section{Introduction}

Regeneration is the process of repairing damaged tissues or replacing lost body parts after injury. The mechanisms that underlie regeneration vary considerably among animal groups and across a wide range of tissue and organ systems. For well over a century scientists have questioned why animals vary in their ability to regenerate (Morgan, 1901). Still, most animal groups, particularly invertebrates (Myohara et al., 1999; Martinez et al., 2005; Agata and Umesono, 2008), have the capacity to regenerate their damaged nervous systems, and much is understood about these processes (Purves and Lichtman, 1985; Moffett, 1996). Furthermore, invertebrate systems like leeches and molluscs provide excellent frameworks for elucidation of the cellular mechanisms underlying the regeneration of specific neural connections (Ready and Nicholls, 1979; Allison and Benjamin, 1985; French and Muller, 1986; Haydon and Kater, 1988; Schacher et al., 1988; Carrow and Levitan, 1989; Liu and Nicholls, 1989; Snyder and Moffett, 1990; Nicholls et al., 1990; Syed et al., 1992; Ross et al, 1994; Zoran and Poyer, 1996; Matsuo et al., 2010). In fact, regenerated networks of neurons of the pond snails Lymnaea and Helisoma exhibit patterns of activity (i.e., fictive behaviors) in vitro that are 
almost indistinguishable from those recorded in intact animals (Syed et al., 1990, 1993). Thus, cell culture approaches are powerful tools for determining cellular mechanisms governing neural regeneration.

In Helisoma, motoneuronal regeneration following nerve crush begins with the growth of numerous processes from the axon that ultimately terminate on potential target cells, both appropriate and inappropriate, where synapse formation takes place (Murphy and Kater, 1978; Bulloch and Kater, 1982; Haydon et al., 1987). Much of the work conducted in this and other molluscan systems has focused on motor systems, especially motoneurons controlling the buccal feeding apparatus and radula (Murphy, 2001; Elliott and Susswein, 2002; Wentzell et al., 2009). Murphy and Kater (1978) demonstrated that after axotomy, motoneurons within the buccal ganglia of Helisoma undergo extensive regeneration and finally reinnervation of their normal target organs. Axonal processes of these neurons are capable of discriminating between appropriate and inappropriate pathways (Murphy and Kater, 1980a), regenerate central connections (Bulloch and Kater, 1982), and accurately reinnervate their peripheral target organs (Murphy and Kater, 1980b). How the reestablishment of central control networks and restoration of peripheral neuromuscular connections are integrated during motor system regeneration remains unclear.

Here, we have examined the mechanisms underlying motoneuronal regeneration of neuromuscular synapses. Helisoma buccal neurons B19 and B110, although activated during different feeding pattern phases, innervate largely the same muscle groups. Motoneuron B19 possesses a synaptogenic nature characterized by the selective formation of neuromuscular synapses (Zoran and Poyer, 1996); that is, it requires specific muscle contact to upregulate its neurosecretory function (Zoran et al., 1990; Poyer and Zoran, 1996). In contrast, neuron B110 is promiscuous in its formation of chemical connections, exhibiting neurotransmission within minutes of contact with a cholinoceptive postsynaptic target (Szabo et al., 2004). Once contacts are formed during regeneration, synapse formation results in differential synaptic properties of the two motoneurons, and physiological analyses suggest the involvement of retrograde target influences on the presynaptic neuronal function. We demonstrate here that ultimately these motoneurons innervate the same muscle groups; however, they do so with varying degrees of synaptic coverage and with variable synaptic efficacy.

\section{Materials and Methods}

\section{Animals}

Experiments were conducted on laboratory stocks of albino (red) pond snails, Helisoma trivolvis (also Planorbella trivolvis Say, 1817), which were maintained in 20-gallon aquaria at $26{ }^{\circ} \mathrm{C}$. Aquaria were kept on a controlled photoperiod of 12-hour light/12-hour dark. Animals were fed lettuce and/or trout chow daily.

\section{Adult buccal ganglia preparations}

Dissection methods have been described previously (Haydon and Zoran, 1991). Two neurons of the buccal ganglia, which innervate radular tensor muscle groups (Kater, 1974; Zoran et al., 1989), were primarily used for these studies: buccal neurons B19 and B110. Snails were de-shelled and pinned to a Sylgard-coated dissecting dish containing Helisoma saline $\left(39.9 \mathrm{mmol} \mathrm{l}^{-1} \mathrm{NaCl}, 1.7 \mathrm{mmol}\right.$ $\mathrm{I}^{-1} \mathrm{KCl}, 4.1 \mathrm{mmol}^{-1} \mathrm{CaCl}_{2}, 1.5 \mathrm{mmol}^{-1} \mathrm{MgCl}_{2}, 10 \mathrm{mmol}$ $1^{-1}$ HEPES, $\mathrm{pH}$ 7.3). Ganglia were excised along with intact buccal nerves and muscles. Buccal motoneurons B19 and $\mathrm{B} 110$ and their connections with the anterior jugalis $(\mathrm{Aj})$ and supralateral radular tensor (Slrt) muscle groups were studied. For neuron B110 recordings, the buccal ganglia were pinned with the rostral side and with the musculature dissected to allow for access to both $\mathrm{Aj}$ and Slrt muscle fibers. Neuron B19 is located caudally, therefore when assessing connectivity in this neuron, the buccal ganglia were pinned with the caudal side exposed. Experiments on isolated buccal ganglia were performed in defined medium (DM). DM consisted of Leibowitz-15 (L-15, Formula No. 82-5154EC, Gibco Laboratories) containing Helisoma salts (mmol l-1: $40.0 \mathrm{NaCl}, 1.7 \mathrm{KCl}, 4.1 \mathrm{CaCl}_{2}, 1.5 \mathrm{MgCl}_{2}$, and 10.0 HEPES at $\mathrm{pH} 7.5$ ).

\section{Regenerating buccal ganglia}

Intact Helisoma were placed in a saturated menthol solution for 15-30 min. Suction was gently applied to the ventral portion of the foot to extract the anterior region of the body from the shell. A dorsal incision was made, and the buccal commissure or the laterobuccal nerves (Lbn) and ventrobuccal nerves (Vbn) nerves associated with the ganglia (bilateral) were crushed with fine forceps. The body wall was sutured, and the animal was allowed from 1 day to 5 weeks to recuperate. Ganglia were then removed from the animals and synaptic connectivity was examined electrophysiologically. Sham operations were also performed in which incisions were made, but nerve trunks were not crushed.

Two points must be noted regarding assessments of postcrush connectivity between neurons B19 and B110. First, for simultaneous electrophysiological recordings, one buccal ganglion was rotated $180^{\circ}$ with respect to the other around the buccal commissure so that both a rostral and caudal side were accessible. Although care was taken not to damage the commissure, some newly forming connections might have been disrupted during this procedure. Second, membrane potential changes produced at distant sites of synapse formation in the commissure were recorded at the 
cell bodies. Therefore, the magnitude of early, transient electrical coupling in these in vivo studies was likely underestimated.

\section{Neuronal cell culture}

Buccal ganglia were isolated from the animal and treated with $0.2 \%$ trypsin (Sigma) for $20 \mathrm{~min}$. Dissection methods for in vitro preparations have been described previously (Haydon and Zoran, 1991). Neurons B19 and B110 were identified and isolated into culture on bovine serum albumin (BSA)-coated 35-mm plastic dishes (Falcon 1008) containing $2 \mathrm{ml}$ of conditioned medium and incubated at $25^{\circ} \mathrm{C}$ (in high humidity) for 2 days. Conditioned medium was prepared by incubating defined medium (DM) with sterile Helisoma central ring ganglia (2 ganglia per milliliter) for 3 days. DM contained 50\% Leibowitz-15 (Gibco) to which L-glutamine $(30 \mathrm{mg} / 100 \mathrm{ml})$, gentamicin $(50 \mathrm{mg} / \mathrm{ml})$, and inorganic salts were added to give final concentrations of 40 $\mathrm{mmol} \mathrm{l}^{-1} \mathrm{NaCl}, 1.7 \mathrm{mmol} \mathrm{l}^{-1} \mathrm{KCl}, 1.5 \mathrm{mmol} \mathrm{l}^{-1} \mathrm{MgCl}_{2}, 4.1$ mmol l-1 $\mathrm{CaCl}_{2}, 10 \mathrm{mmol} \mathrm{l}^{-1}$ HEPES, pH 7.4.

\section{Buccal muscle dissociation}

The Slrt and Aj muscle groups were dissected from the buccal mass and sterilized through three washes of Helisoma antibiotic saline (sterile water containing Helisoma salts and $150 \mathrm{mg} / \mathrm{ml}$ gentamicin) and placed into a sterile, 5 -ml glass test tube pre-coated with BSA. Muscles were dissociated into individual fibers through incubation in DM containing $2 \mathrm{mg} / \mathrm{ml}$ collagenase/dispase (Boehringer-Mannheim) at room temperature for $12 \mathrm{~h}$ in a $32{ }^{\circ} \mathrm{C}$ water bath. After incubation, DM was added to the test tube, and muscle fibers were fully dissociated using gentle agitation and centrifugation $(1000 \times g$ for $5 \mathrm{~min})$. Fibers were then collected and transferred to a BSA-coated plastic culture dish (Falcon 1008). Subsequently, the individual muscle fibers were transferred into poly-L-lysine (PLL)-coated 35-mm dishes and plated into culture on opposite sides of a neuronal cell body.

\section{Electrophysiological techniques}

Electrophysiological analyses were performed using intracellular recording techniques. Glass microelectrodes (borosilicate; FHC) were filled with $1.5 \mathrm{~mol} \mathrm{l}^{-1} \mathrm{KCl}$, and possessed tip resistances ranging from 10 to $20 \mathrm{M} \Omega$. Current-clamp recordings of neuronal membrane potentials were amplified using bridge-balanced electrometers (Getting Instruments or World Precision Instruments). Simultaneous recordings were made from cell pairs or from neuronmuscle preparations. For in situ recordings, ganglia were bathed in high-calcium $\left(41 \mathrm{mmol} \mathrm{l}^{-1} \mathrm{CaCl}_{2}\right) \mathrm{DM}$ to reduce feeding motor-pattern activity and other synaptic inputs to muscle fibers. Preparations were visualized with a dissect- ing microscope (Olympus). Neuronal membrane potential was maintained with base current injection at approximately $-70 \mathrm{mV}$, and muscle membrane potential was maintained at approximately $-50 \mathrm{mV}$. Electrical coupling was measured by injection of constant-amplitude, hyperpolarizing current pulses (0.5-1 nA pulse for 3 s) into one neuron, while membrane voltage changes in both the presynaptic (injected) neuron and its postsynaptic (noninjected) partner were monitored. In some experiments neurons were injected with the fluorescent dye Lucifer yellow (Sigma) to examine neuronal architecture and verify cell identity.

The neuron-muscle chemical connection, as well as the chemical connection from neuron B110 onto neuron B19, was studied by injection of depolarizing current into presynaptic neuron B110. Four to five trains of action potentials (1 per second) were evoked, and postsynaptic potentials (PSPs) were examined. The presence or absence of PSPs and the location of the fiber from which the recording was made within each identified muscle group were used to generate topographical synaptic maps. Electrophysiological recordings were digitized by a PowerLab A/D data acquisition system linked to an Apple computer using Chart software (AD Instruments, ver. 4.1.1).

For in vitro assessments of connectivity, isolated neurons were incubated for 2 days in nonadhesive conditions and then transferred between parallel grooves scored onto the bottom of PLL-coated 35-mm dishes (Falcon 3001). These grooves restricted neuritic outgrowth to the area between the etched parallel lines. Aj and Slrt muscle fibers were then manipulated into the culture dishes on opposite sides of the neuron and plated within 50-100 $\mu \mathrm{m}$ of the cell body. After an additional 3 days of co-culture, an ACh-sensitive assay cell was manipulated into contact with the neurites of the neurons. Assay cells were neurons cultured as spheres for 2 days on BSA-coated dishes containing $2 \mathrm{ml}$ of conditioned medium. These spherical B19 somata are highly cholinoceptive and function as ACh-sensors detecting neurotransmitter release from presynaptic neurites (Haydon and Zoran, 1989, 1991). Neurite-assay contacts were established $30 \mathrm{~min}$ to $1 \mathrm{~h}$ prior to the assessment of ACh release. Assay cells were positioned just proximal to sites of neuritemuscle contact.

\section{Quantal analysis of synaptic transmission}

The amplitudes of miniature postsynaptic potentials (mPSPs) were measured over a period of $2 \mathrm{~min}$. The amplitudes of the evoked PSPs were then measured during a concomitant current injection of the presynaptic neuron to elicit trains of action potentials. The assay cell membrane potential was maintained with a base current injection at $-80 \mathrm{mV}$. There were approximately $50-100 \mathrm{mPSPs}$ and 200-400 PSPs measured for each preparation. The amplitudes were measured from baseline to the peak of the 
synaptic potential. To determine quantal size, a model cell preparation was used, and the number of occurrences and their amplitudes of the mPSP were plotted. The amplitude of the most frequent mPSP was used as an estimate of quantal size.

\section{Neuronal track tracing}

Whole-mount preparations were pinned out on Sylgard blocks in Helisoma saline. Individual identified neurons were injected via micropipettes containing a 3\% Lucifer yellow/fast green solution by using a picospritzer (General Valve). Following varying periods of diffusion (2-24 h), preparations were fixed and processed for imaging. In some experiments, preparations were embedded, and cryosections were cut and mounted on glass slides. Preparations were imaged using an Olympus inverted microscope and Simple PCI software (Hamamatsu, ver. 6.0) and imported into Adobe Photoshop for post-capture processing.

\section{Data analysis}

Statistical analyses were performed using Statview software (Abacus Concepts, Inc., ver. 4.5). The Student's $t$-test was used for comparison between two populations. For analyzing multiple populations, an analysis of variance was employed, and comparisons were made using Fisher's paired least significant difference test. Data is presented as a mean \pm SEM unless otherwise stated. Statistical significance was represented as $P<0.05$.

\section{Results}

Connectivity of the Helisoma buccal neuromuscular system

Bilaterally symmetrical motor neurons, located in the paired buccal ganglia in Helisoma, control the feeding musculature and associated structures: the radula, odontophore, salivary glands, and esophagus. Like other invertebrate motor systems, the buccal musculature of Helisoma is innervated by both motor and modulatory neurons. For example, cerebral neuron $1(\mathrm{C} 1)$ is a serotonergic neuron that projects through the cerebral-buccal connective to innervate the buccal ganglion, but also projects via buccal nerves to the buccal musculature (Fig. 1A). Stimulation of C1 elicits rhythmic firing in buccal neurons and increases the strength of contractions of radular muscles (Murphy, 2001; Fig. 1B). Several aspects of the regeneration of buccal motor neurons are differentially regulated by serotonin (Murphy et al., 1985; McCobb et al., 1988; Murrain et al., 1990; Price and Goldberg, 1993). In the present studies of buccal neuromuscular regeneration, we have limited our analysis to buccal retractor neuron $\mathrm{B} 110$, which fires during the $\mathrm{S} 2$ phase of the motor pattern, and hyper-retractor neuron B19, which fires during the $\mathrm{S} 3$ phase (Fig. 1C). Bursts of action poten- tials in these motor neurons drive summating excitatory junction potentials (EJPs) in specific sets of muscle fibers. For example, spikes in B19 evoke EJPs in muscle fibers of the supralateral radular tensor (Slrt) muscle (Fig. 1D). Interestingly, although neurons B19 and B110 fire out of phase during rhythmic motor outputs and are involved in different muscle movements, they possess axonal projections that terminate onto similar sets of buccal muscles, which regulate the movements of the radula and odontophore.

The axons of buccal motor neurons responsible for delivering rhythmic excitation to neuromuscular synapses are, in part, located in the Vbn and Lbn. For example, neuron B19 possesses two initial axons extending from the cell body, one that projects ipsilaterally (Fig. 2A) and one that projects contralaterally. Both of these axons then bifurcate, with one branch connecting to buccal muscles by way of the Vbn and the other by way of the Lbn. At the buccal muscle, the axon branches multiple times to terminate in a meandering chain of synaptic boutons (Fig. 2B, C). In this way, each motoneuron (e.g., B19) is responsible for the excitation and contraction of hundreds of individual cells within the fiber bundles of specific muscle groups (e.g., the Slrt).

\section{Neuromuscular connectivity of neurons in the Helisoma motor system}

Kater (1974) originally described the peripheral connectivity of motoneuron B19 to muscles of the buccal mass. As originally described by Kater, and as seen in Figures 1 and 2, one of the muscle targets of B19 is the Slrt muscle group. However, a rigorous examination of innervation patterns, both ipsilateral and contralateral, for retractor and hyperretractor motoneurons has not been performed. Therefore, we simultaneously recorded from identified motoneurons in the buccal ganglia as well as from the potential muscle targets for each in the buccal mass, while stimulating bursts of action potentials in the motoneuron using intracellular current injection. The major muscle targets investigated are illustrated in Figure 3A. Stimulus-evoked EJPs were measured for each muscle fiber penetration, and the percentage of muscle recordings with detectable EJPs was documented. As expected, neuron B19 innervated the Slrt muscles, and similar patterns of connectivity were present in both ipsilateral and contralateral muscle bundles (Fig. 3B; $n=3-6$ animals per muscle group). Neuron B19 also innervated the supramedial radular tensor (Smrt) and inframedial radular tensor (Imrt) muscle groups bilaterally, with essentially equal functional synaptic coverage (Fig. 3B; $n=3-6$ animals per muscle group). Similar observations have been made in Aplysia, where buccal motoneurons innervate multiple muscle groups of the buccal mass (Jordan et al., 1993). Consid- 


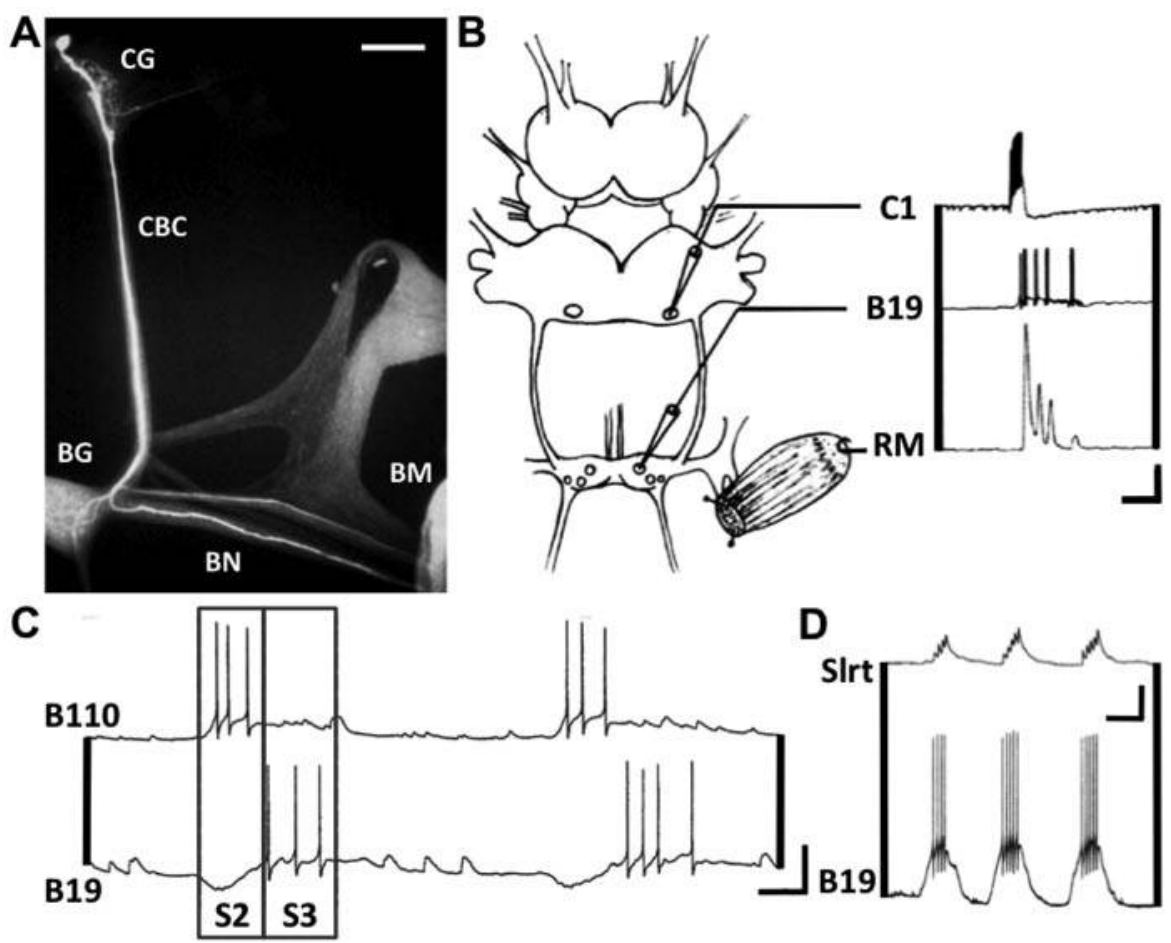

Figure 1. Neural organization of the Helisoma buccal motor system. (A) The cell body of neuron $\mathrm{C} 1$ of the cerebral ganglion (CG) is visualized by injection with Lucifer yellow. The axon of $\mathrm{C} 1$ projects through the cerebrobuccal connective $(\mathrm{CBC})$ and branches to innervate targets in the buccal ganglion (BG) and the buccal mass (BM), the latter through the buccal nerves (BN). Horizontal scale bar equals $180 \mu \mathrm{m}$. (B) Stimulation of $\mathrm{C} 1$ activates rhythmic firing in buccal motor neurons and is associated with contractions of the radular musculature (RM). Vertical scale bar equals $40 \mathrm{mV}$. Horizontal scale bar equals $5 \mathrm{~s}$. (C) Activity of neurons B19 and B110 is not in phase during serotonin-evoked rhythmic firing patterns. B110 functions during the S2 phase (i.e., fictive retraction of the radula), while B19 functions during the $\mathrm{S} 3$ phase (i.e., hyper-retraction of the radula). Vertical scale bar equals $30 \mathrm{mV}$. Horizontal scale bar equals $50 \mathrm{~ms}$. (D) B19 action potentials activate one-for-one, summating EJPs in Slrt muscle fibers. Vertical scale bar equals $30 \mathrm{mV}$ (top trace) and $20 \mathrm{mV}$ (bottom trace). Horizontal scale bar equals $100 \mathrm{~ms}$.

erable connectivity between neuron B19 and the Aj muscle of the buccal mass outer wall was detected on both sides of the feeding structure. This was surprising because we had not observed obvious contractions of the Aj following B19 stimulation. In fact, B19-evoked contractions of the $\mathrm{Aj}$ muscle were almost undetectable, suggesting less efficacious excitation-contraction coupling.

Peripheral connectivity of neuron B110 was also examined, and several features of its innervation pattern differed from those of B19. Neuron B110 was connected to each of the muscle groups tested (the Imrt was not examined), but its synaptic coverage of the Slrt muscle group was less extensive than its connectivity to the Aj muscle group (Fig. $3 \mathrm{C} ; n=3-6$ animals per muscle group). Furthermore, in contrast to the neuromuscular effectiveness of B19, neuron $\mathrm{B} 110$ elicited robust contractions in the $\mathrm{Aj}$, but only weak responses in the Slrt. In a rare case of bilateral asymmetry in innervation by these motoneurons, B110-evoked EJPs were detected in the ipsilateral, but not the contralateral, Smrt muscle group.
Synaptic connections between B19 and B110 form after nerve crush

We used electrophysiological recordings identical to those previously employed for assessment of adult motoneuronal connectivity to assess synaptic regeneration after axonal damage by nerve crush. We should point out that neuron B19 is known to form electrical synapses both ipsiand contralaterally with other motor neurons in the buccal ganglia, and these connections have been well documented (Kater, 1974; Kaneko et al., 1978; Haydon and Kater, 1988; Szabo et al., 2004). Neurons B19 and B110 do not possess electrical or chemical synaptic connectivity with each other in adult ganglia (Fig. 4A; $n=6$ preparations). In Helisoma, nerve crush and axonal regeneration result in the formation of many novel neuronal (central) connections, both electrical and chemical (Hadley et al., 1982). Following nerve crush and axotomy in the present studies, transient electrical and chemical connections form between motoneurons B19 and $\mathrm{B} 110$ (Fig. 4B; $n=6$ preparations). The timing of 


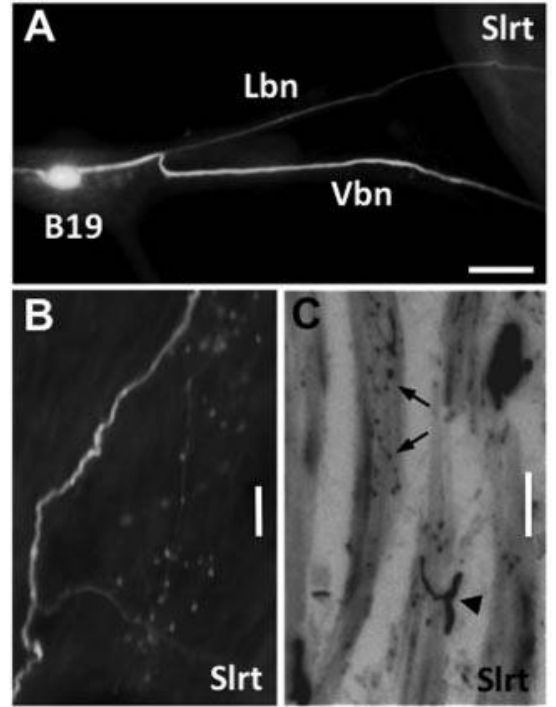

Figure 2. Neuromuscular structure of Helisoma buccal motoneurons. (A) Lucifer yellow injection of buccal neuron B19 illustrates the two ipsilateral axons that project to muscles of buccal mass, such as the Slrt. Two buccal nerves, the Lbn and Vbn, connect buccal motoneurons to some of the radular muscles. Horizontal scale bar equals $180 \mu \mathrm{m}$. (B) Lucifer yellow-filled axons of B19 terminate in the Slrt in chains of synaptic boutons (fluorescent puncta) that innervate many individual muscle fibers. Vertical scale bar equals $10 \mu \mathrm{m}$. (C) Frozen section of Slrt muscle fibers illustrating the dye-filled synaptic terminals of neuron B19 (arrows). The arrowhead indicates a B19 axonal branch point. Vertical scale bar, $10 \mu \mathrm{m}$.

electrical and chemical synapse formation of these neurons was staggered, such that electrical connections emerged within the first days after nerve crush and were largely undetectable by day 7 post-crush (Fig. 4C). In contrast, the chemical connection between B110 and B19 did not reach peak strength until transient electrical connectivity was diminished. This chemical connection is unidirectional from B110 (presynaptic neuron) to B19 (postsynaptic neuron) and is inhibitory (Szabo et al., 2004). Furthermore, the chemical connection persisted for weeks following nerve injury (Fig. 4C).

It should be noted that the recording of this chemical synapse in Figure 4B was conducted with $\mathrm{KCl}$ electrodes, which reverse the polarity (i.e., direction of charge movement) of the underlying synaptic current. Thus, although the record appears to indicate an excitatory connection, it is in fact inhibitory (Szabo et al., 2004). Therefore, in response to nerve injury, neurons B19 and B110 form two synaptic connections: first, a bidirectional electrical connection that is strongest on day 2 post-crush and weakens by day 7 ; second, a unidirectional inhibitory connection from B110 onto B19 that forms on day 6 post-crush and does not diminish in strength for many weeks. We will see in the next section that the transition between central electrical and chemical synapse formation by motoneurons occurs at the time that regenerating axons are first contacting the peripheral buccal musculature.

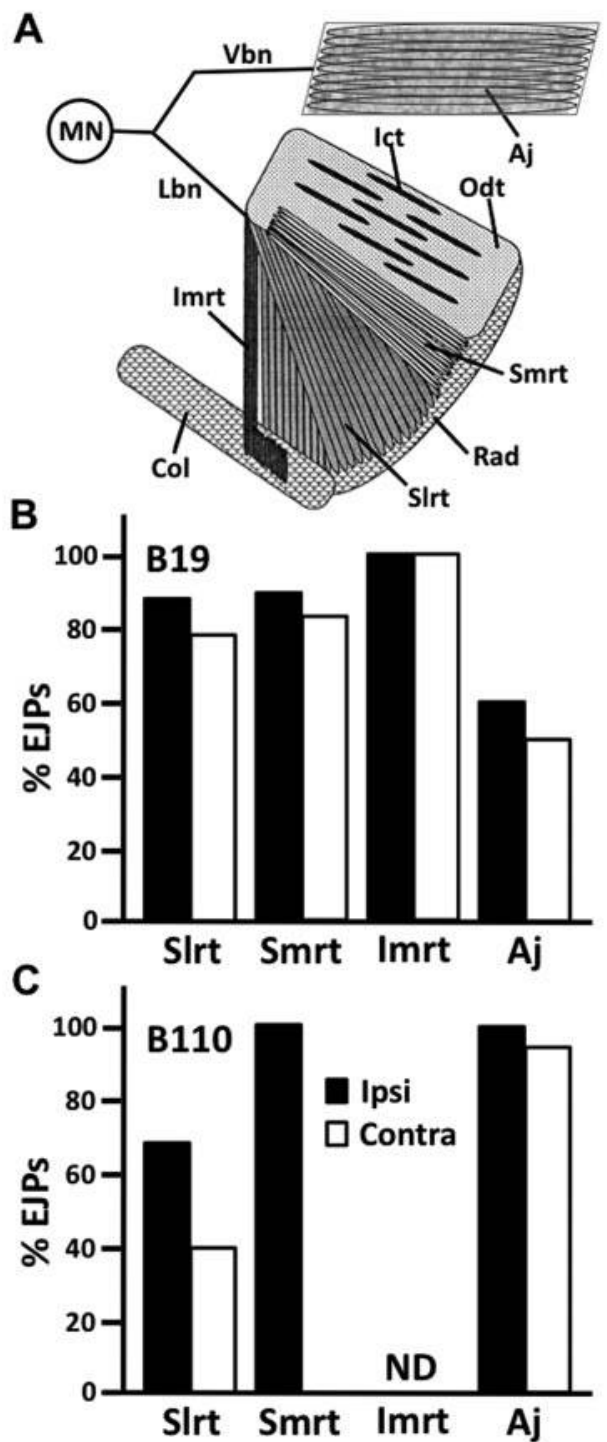

Figure 3. Neuromuscular connectivity of buccal motor neurons B19 and B110. (A) The buccal muscles innervated by these motoneurons (MN) are indicated in this illustration of a dissected and flattened feeding musculature. Vbn, ventrobuccal nerve; Lbn, laterobuccal nerve; Col, collostyle; Imrt, inframedial radular tensor; Slrt, supralateral radular tensor; Rad, radula; Smrt, supramedial radular tensor; Ict, intracartilage tensor; Odt, odontophore; Aj, anterior jugalis. (B) B19 axons project both ipsilaterally and contralaterally to innervate muscles on both sides of the buccal mass. Vertical bars equal the percentage of muscle fibers where intracellular recordings detected B19-evoked EJPs. Solid bars indicate ipsilateral muscles and open bars indicate contralateral muscles. (C) B110 also innervates muscles on both sides of the buccal mass. Vertical bars equal the percentage of muscle fibers where EJPs were detected. ND, no data collected. $n=$ 3-6 preparations per muscle group.

\section{Neuron B19 regenerates peripheral connectivity accurately and rapidly}

Like many other invertebrate animal groups, molluscs regenerate their nervous systems quickly and accurately 


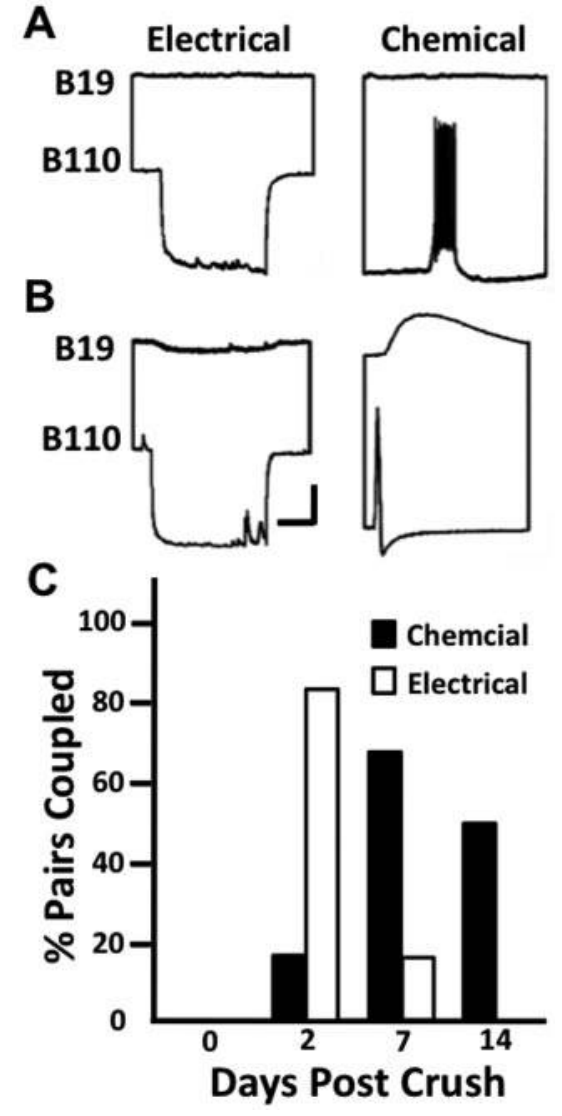

Figure 4. Transient electrical and chemical synaptic connections between B110 and B19. (A) Electrophysiological records demonstrate the lack of electrical and chemical connections between B19 and B110 in intact buccal ganglia ( $n=6$ preparations). Vertical scale bar equals $10 \mathrm{mV}$ (top trace) and $20 \mathrm{mV}$ (bottom trace). Horizontal scale bar equals $1 \mathrm{~s}$ (left panel) and $100 \mathrm{~ms}$ (right panel). (B) Electrophysiological records represent both transient electrical coupling (day $2 ; n=6$ preparations) and transient chemical synaptic connections (day 7;n=6 preparations) in adult Helisoma buccal ganglia during regeneration following nerve crush. Vertical scale bar equals $10 \mathrm{mV}$ (top trace) and $20 \mathrm{mV}$ (bottom trace). Horizontal scale bar equals $1 \mathrm{~s}$ (left panel) and $50 \mathrm{~ms}$ (right panel). (C) Vertical bars indicate the percentage of neuronal pairs where dual intracellular recordings detected chemical (solid bars) and electrical (open bars) connections in the days following nerve crush ( $n=5-7$ preparations per data point).

after injury (Moffett, 1996). Buccal motoneurons specifically can reconnect to peripheral targets within one week. For example, functional reinnervation of the salivary gland by motoneuron B 4 occurs by day 7 after axotomy (Murphy and Kater, 1980b). We have determined the timing and fidelity of neuromuscular synapse regeneration by buccal motoneuron B19. These experiments used both sham-operated animals (defined as day 0 controls) and animals in which the axons of this neuron were severed by bilateral crush of both the Lbn and Vbn, the two nerves that connect the buccal ganglia to the buccal musculature (Figs. 2A and $3 \mathrm{~A})$. After 3 days to allow for axonal regeneration to the peripheral musculature, assays were performed to detect the presence of synaptic neurotransmission.

Motoneuron-evoked EJPs at B19-Slrt synapses were first detected in the ipsilateral musculature on day 6 after crush of the buccal nerves. This functional reinnervation was initiated at the point of entry of the growing axon into the fanlike muscle group, with all other Slrt muscles lacking evoked EJPs on that day (Fig. 5A). The first regenerating axons to enter into the Slrt bundles, with branches terminating in large bouton-like endings between days 3-5 postcrush (Fig. 5B; $n=6-7$ animals per day post-crush), a time when evoked EJPs were not detected (Fig. 5C). Once evoked EJPs were present, their efficacy was typically not one-for-one during day 6-8 post-crush. Rather, many action potentials in a train failed to elicit postsynaptic potentials (Fig. 5D). Only after 9-11 days of regeneration did most preparations exhibit virtually complete functional reinnervation (Fig. 5A) and one-for-one B19-Slrt neuromuscular synaptic efficacy (Fig. 5E; $n=6-7$ animals per day post-crush).

Prior to B19-Slrt recovery of function after day 6 postcrush, spontaneous or miniature excitatory junction potentials (mEJPs) were detected in muscle fibers. These are considered spontaneous due to the fact that no action potentials in B19 were elicited to generate these synaptic events. Interestingly, mEJPs are extremely rare in adult Slrt muscle fibers, but were detected in greater than $80 \%$ of muscle fibers after 3-5 days of buccal nerve regeneration (Fig. 5F; $n=6-7$ animals per day post-crush). This elevated occurrence of mEJPs was transient and rapidly declined between days 6 and 11 post-crush (Fig. 5F). This decline in spontaneous neuromuscular synaptic events coincided with a marked increase in the incidence of evoked EJPs recorded in these regenerating motor systems (Fig. 5G; $n=6-7$ animals per day post-crush). Therefore, these studies defined three phases of Helisoma neuromuscular regeneration. First, there was an initial period of axonal emergence into the muscle group that was associated with spontaneous neurotransmitter release, but no evoked release (Phase 1; Fig. 5A). Second, an intermediate period of regeneration emerged that lasted from days 6 to 8 , in which spontaneous events became rare and an evoked release of neurotransmitter, although not efficacious, was present (Phase 2; Fig. 5D). Third, by 9-11 days post-crush, functional recovery of efficacious neuromuscular synaptic transmission was largely achieved, where Slrt EJPs were detected in a one-for-one fashion in response to B19 action potentials in approximately $80 \%$ of muscle fibers (Phase 3; Fig. 5E, G).

The previous description of B19-Slrt neuromuscular regeneration was presented with respect to the ipsilateral musculature. Identical experiments were conducted on the contralateral Slrt muscle, and the results were virtually identical. Therefore, these analyses are not presented here. 


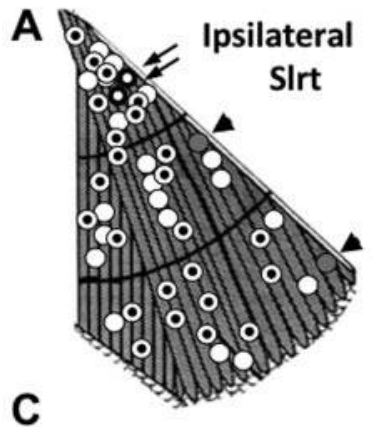

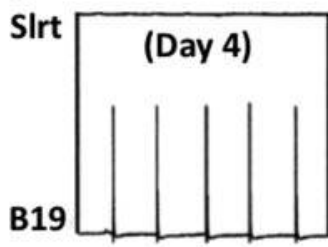

Phase 1

$\mathbf{F}$

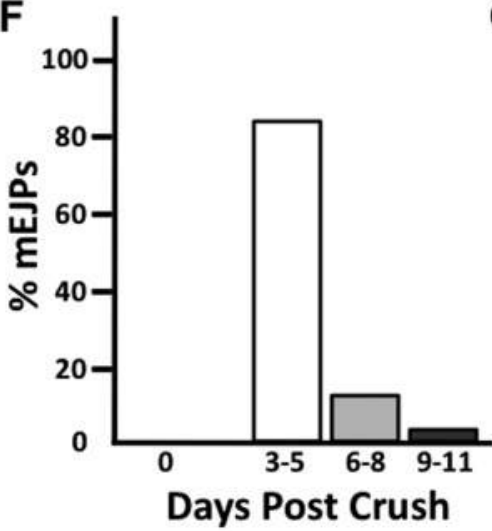

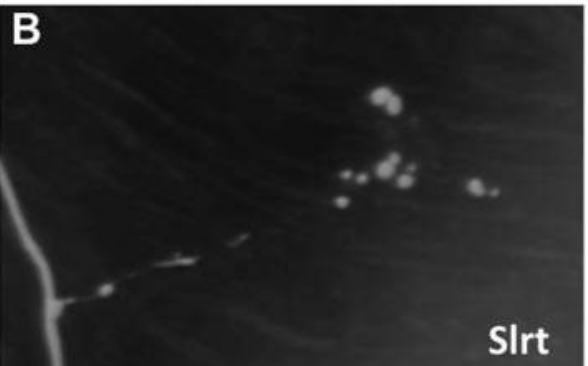

D
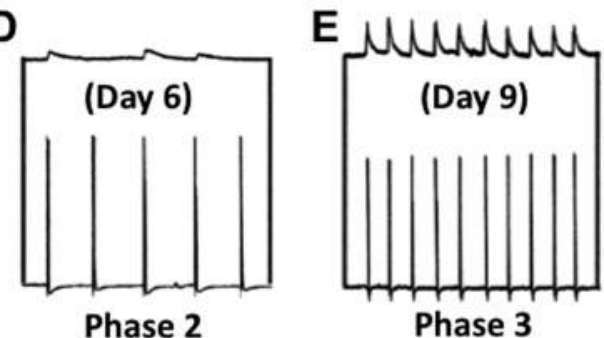

Phase 3

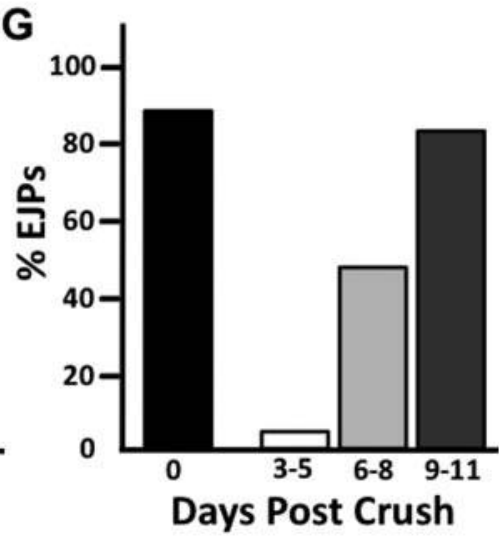

Figure 5. Phases of B19-Slrt neuromuscular synapse regeneration. (A) Open circles illustrate the absence of evoked neuromuscular synaptic transmission on day 2 post-crush. By day 6 (partially open circles, indicated by arrows), only two muscle fibers near in at the base of Slrt fan-shaped muscle group possess B19-evoked EJPs. Almost all muscle fibers (partially closed circles, black dots in center) at day 9 post-crush are innervated by B 19 . Only two fibers on this day (gray circles, indicated by arrowheads), at the extreme edge of the muscle, were apparently not innervated by B19. (B) Initial reinnervation of the Slrt by B19 is demonstrated by Lucifer yellow-filled motoneuronal terminals on day 5 post-crush. (C) Phase 1 neuromuscular synapse formation is characterized by the lack of B19-evoked EJPs in the Slrt muscle. (D) Phase 2 synapse formation involves the elicitation of weak EJPs in Slrt fibers. These events are not coupled one-for-one with B19 action potentials. (E) Phase 3 synapse formation is characterized by strong, one-for-one EJPs. (F) Vertical bars indicate the percentage of Slrt muscle fibers where dual intracellular recordings detected mEJPs in the days following nerve crush $(n=$ 6-7 preparations per day post-crush). (G) Vertical bars indicate the percentage of Slrt muscle fibers where EJPs were detected. Day 0 represents intact control preparations $(n=4$ preparations).

The only observed difference in these studies was that spontaneous mEJPs were still detected in greater than $50 \%$ of contralateral Slrt muscle fibers by $6-8$ days post-crush, and less than $40 \%$ of muscle fibers exhibited evoked EJPs by days $9-11$ post-crush. Taken together, three phases of neuromuscular regeneration are also present in contralateral musculature, but this regeneration appears to be delayed by 3 days relative to ipsilateral muscles. Similar recordings were made from other muscle groups during functional recovery. Although these groups were not rigorously investigated, they showed phases of neurouscular regeneration similar to those observed during B19-Smrt and B19-Aj recordings. The timing of their functional reinervation was also similar and in some instances more rapid (data not shown).

\section{Differential efficacy of buccal neuromuscular synapses}

As demonstrated in the previous studies, neurons B19 and B110 each innervate the Slrt and Aj muscle bundles in vivo; however, as previously mentioned, neuromuscular ex- 
A
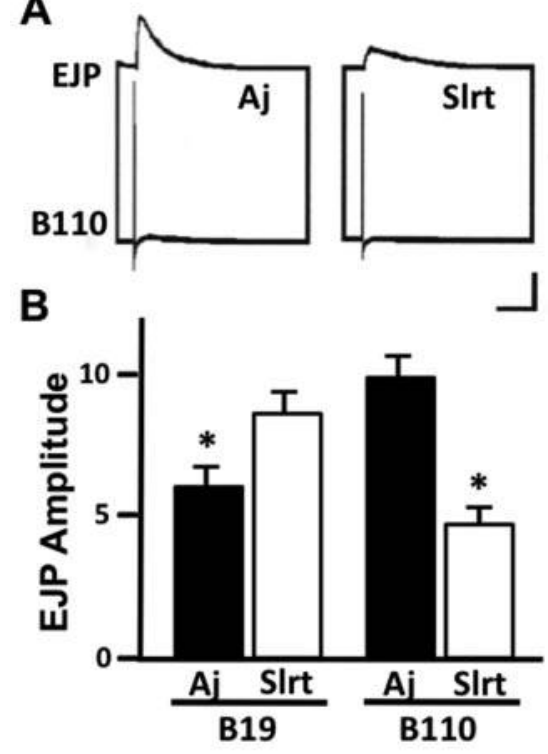

Figure 6. B19 and B110 neuron-muscle synaptic strength. (A) In vivo recordings from neuron B110 show a stronger postsynaptic EJP in an $\mathrm{Aj}$ muscle fiber than in the Slrt. Vertical scale bar equals $10 \mathrm{mV}$ (top trace) and $20 \mathrm{mV}$ (bottom trace). Horizontal scale bar equals $100 \mathrm{~ms}$. (B) Neuron B19 evoked significantly greater amplitude EJPs in Slrt muscle fibers $(n=$ 32 preparations) than in $\mathrm{Aj}$ fibers $(n=37$ preparations; *, $P<0.05)$. Neuron B110 elicited EJP amplitudes at Aj muscle fibers ( $n=40$ preparations) that are significantly greater than those in Slrt fibers $(n=41$ preparations; *, $P<0.05$ ).

citation at B19-Aj and B110-Slrt connections was not associated with strong muscle contractions. To determine whether these weak muscle contractions were associated with a reduction in synaptic efficacy, we performed quantitative electrophysiological analyses of EJP amplitudes. Dual electrophysiological recordings demonstrated differences in amplitude of EJPs between $\mathrm{Aj}(n=37$ preparations) and Slrt ( $n=32$ preparations) muscle fibers after stimulation of neuron B19 (Fig. 6A); that is, EJP amplitudes in Slrt muscle fibers were significantly larger than EJPs evoked in $\mathrm{Aj}$ muscle fibers (Fig. 6B). In contrast, presynaptic stimulation of neuron B110 induced EJPs of significantly greater amplitude in $\mathrm{Aj}(n=40$ preparations) muscle fibers than in Slrt ( $n=32$ preparations; Fig. 6B).

\section{Target-induced regulation of motoneuronal synaptic efficacy}

To determine whether the mechanism underlying differences in synaptic efficacy involved neuronal changes due to muscle-derived influences, buccal motoneurons and muscle fiber targets were isolated into cell culture and manipulated into positions that ensured regeneration of neuromuscular contacts. Neurons were plated between grooves scored onto the cell culture dish to restrict neurite outgrowth and facil-
A
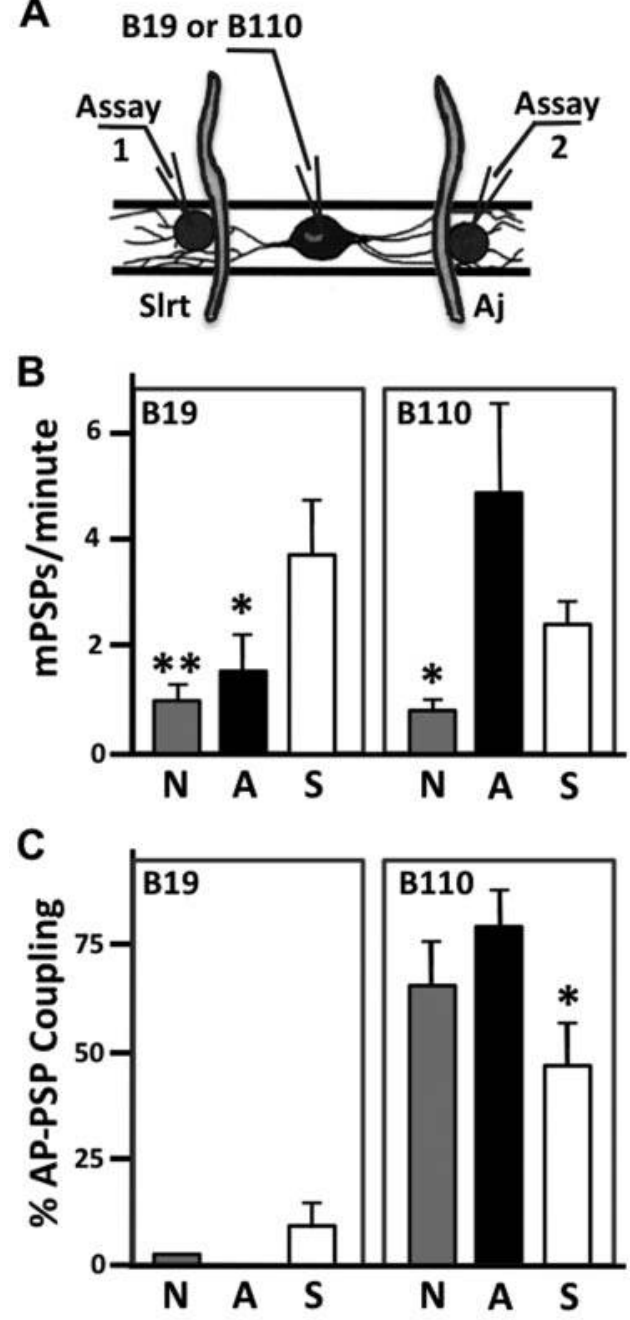

Figure 7. Assessment of motoneuron secretory function in cell culture. (A) An illustration of the in vitro neuromuscular-assay preparation indicates the two ACh-sensitive assay cells used to detect motoneuronal synaptic transmission at neuromuscular contacts. The culture dish was scored to limit outgrowth to the area between the grooves, directing neurites toward muscle targets. (B) B19 and B110 mPSP rates were assessed with no muscle contact $(\mathrm{N} ; n=14$ [B19] and 12 [B110] preparations); $\mathrm{Aj}$ contact (A; $n=11$ [B19] and 18 [B110] preparations); and Slrt contact (S; $n=10$ [B19] and 19 [B110] preparations). B19 rates were significantly greater following 2 days of Slrt contact (**, $P<0.01$; *, $P<$ $0.05)$. B110 rates were significantly greater following 2 days of $\mathrm{Aj}$ contact $(*, P<0.05)$. (C) The percentage of assay cells with B19- and B110evoked PSPs was assessed with no muscle contact $(\mathrm{N} ; n=14$ [B19] and 12 [B110] preparations), Aj contact (A; $n=11$ [B19] and 18 [B110] preparations) and Slrt contact (S; $n=10$ [B19] and 19 [B110] preparations). B19 AP-PSP coupling was weak in all conditions. B110 AP-PSP coupling was significantly lower in Slrt muscles as compared to those in $\mathrm{Aj}$ muscles $(*, P<0.05)$.

itate growth cone interactions with muscle fibers (Fig. 7A). To isolate mechanisms related to the presynaptic neuron from possible alterations in postsynaptic responsiveness, ACh-sensitive assay cells were placed at sites of neuritemuscle contact to monitor ACh release from these cholin- 
ergic motoneurons. ACh-sensitive assay cells are well-defined tools for monitoring transmitter release from buccal neurons (Haydon and Zoran, 1989, 1991; Zoran et al., 1990). To assess the rate of spontaneous ACh release from each neuron, mPSPs were recorded. Neurites of both neurons B19 and B110 possessed higher mPSP rates following contact with Slrt $(n=10, \mathrm{~B} 19$ and $19, \mathrm{~B} 110)$ and $\mathrm{AJ}(n=$ 11, B19 and 18, B110) muscle fibers as compared to neurites of neurons alone $(n=14, \mathrm{~B} 19$ and $12, \mathrm{~B} 110)$ in culture (Fig. 7B). However, neuron B19 exhibited a significantly higher mPSP rate at Slrt-neurite contacts than at $\mathrm{Aj}$-contacted neurites (Fig. 7B). In contrast, neurites of B110 contacting $\mathrm{Aj}$ muscle fibers possessed higher rates of $\mathrm{mP}-$ SPs than those of neurites contacting Slrt fibers (Fig. 7B).

Unlike neuron B19's requirement for an appropriate target for the regeneration of functional synapses (Zoran et al., 1996; Zoran and Poyer, 1999), neuron B110 is able to form chemical synapses when contacted by any cholinoceptive target (Fig. 7C). Action potential-evoked postsynaptic potentials (AP-PSPs) recorded with ACh-reporter cells were monitored in dual muscle co-cultures. Although neuron B110 does not require contact with a muscle target to acquire ACh secretory capability, neurites of B110 contacting Slrt muscle fibers exhibited less efficacious release of ACh than those contacting Aj muscle fibers (Fig. 7C). This result indicates a retrograde influence of the muscle fiber on presynaptic mechanisms of $\mathrm{ACh}$ release in motor neuron B110. In contrast, B19 has minimal synaptic efficacy in these cultures, with or without muscle contact. B19 AP-PSP coupling was approximately $10 \%$ following interaction with Slrt muscle fibers only (Fig. 7C).

\section{Retrograde regulation of presynaptic release properties}

We hypothesized that differences in motoneuronal synaptic efficacy at neurites contacting different muscle targets were mediated by changes in presynaptic properties, since PSPs were recorded with assay cells characterized by consistent ACh-sensitivity (Haydon and Zoran, 1991). Additionally, muscle target contact is known to alter action potential-dependent calcium accumulation in presynaptic neurites of buccal neurons (Zoran et al., 1993; Funte and Haydon, 1993). Therefore, the probability of vesicular release may be altered at neuritic sites of muscle targets in a neuron-specific fashion. To test this hypothesis in neuron B19 would be difficult because synaptic efficacy in these neurons is very low in cell culture (Fig. 7C). Consequently, we analyzed the secretory properties of the more efficacious neuron B110 during regeneration in cell culture. It should be noted that quantal analyses were conducted with assay cells to again avoid differences in postsynaptic receptor density or sensitivity to ACh.

Since the rate of miniature excitatory synaptic potentials for neuron B110 was elevated following contact with $\mathrm{Aj}$ muscle fibers in culture, we tested whether quantal size was also affected by Aj muscle. Neuron B110 co-cultured with $\operatorname{Aj}(n=18$ preparations) and Slrt $(n=19$ preparations $)$ muscle fiber contacts again showed elevated rates of mPSPs only at sites of contact with $\mathrm{Aj}$ muscle fibers (Fig. 8A). However, mean mPSP amplitudes were not significantly different between $\mathrm{Aj}$ and Slrt-contacted neurites $(P=0.25$; Fig. 8C). Thus, even with a more robust frequency of spontaneous release at $\mathrm{Aj}$ contacts, there was no change in the amount of neurotransmitter released per vesicle, as indicated by the voltage change associated with one spontaneous release event (quantal size).

Since quantal size was not different between $\mathrm{Aj}$ and Slrt-contacted neurites, we next assessed evoked PSP amplitudes as a measure of the amount of neurotransmitter (i.e., level of exocytosis of synaptic vesicles) per action potential at neurite-muscle contact sites for neuron B110. To achieve this, trains of action potentials were stimulated presynaptically while simultaneously recording postsynaptic responses in an assay cell contacting B110 neurites (Fig. 8B). Neuron B110 exhibited efficacious release of ACh at sites of contact with both Slrt and Aj muscle fibers; however, Aj-contacted neurites possessed significantly greater PSP amplitudes than Slrt-contacted neurites (Fig. 8D). Several features of the PSPs elicited in ACh reporter cells suggested enhanced abilities for synaptic transmission of Aj-contacted neurites. First, PSPs maintained consistently higher amplitudes during high-frequency stimulation than did Slrt-contacted neurites. Second, the slope of the rising phase of PSPs was consistently greater at Aj-contacted neurites. Finally, estimates of quantal content at $\mathrm{Aj}$-contacted neurites, calculated as the sum of the mean PSP amplitude divided by the mean mPSP amplitude, were significantly different than those at Slrt-contacted processes (Fig. 8E). Taken together, these results support a mechanism of retrograde regulation of presynaptic release properties by muscle targets during neuron B110 neuromuscular regeneration.

\section{Discussion}

A fundamental difference between vertebrate and invertebrate motor systems is the nature of their neuromuscular innervation. Most invertebrate muscle fibers, including those of the Helisoma buccal musculature, receive synaptic inputs from multiple motoneurons (for example, B19, B110, and many others) and modulatory neurons (for example, C1), such that significant motor plasticity can be generated by neuromuscular synapses in the periphery (Heyer et al., 1973). Vertebrate skeletal muscles, on the other hand, typically have motoneuronal input from a single spinal motoneuron, and most modulation occurs centrally. B19 and B110 are active during different phases of fictive Helisoma feeding behavior, and the phases of activation between these 
A
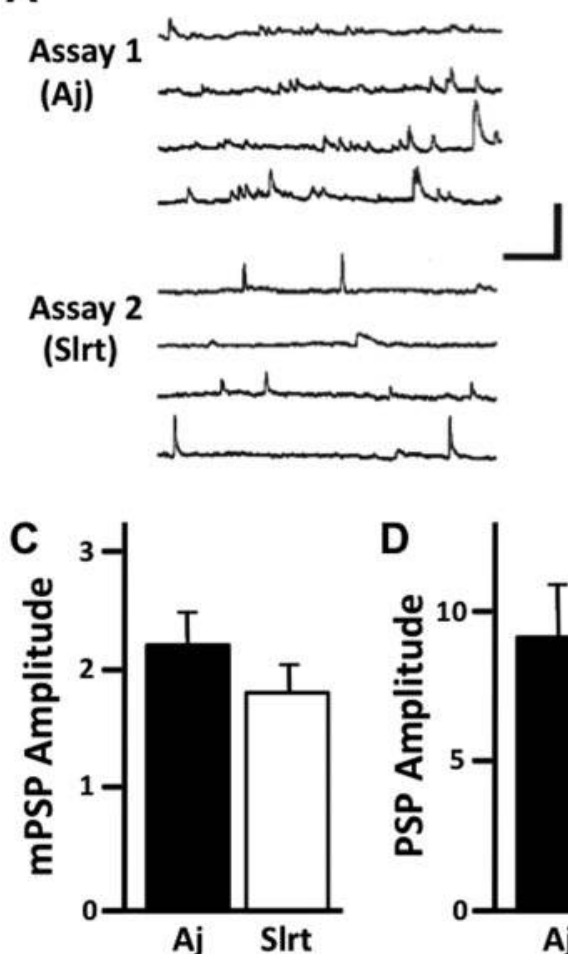

B

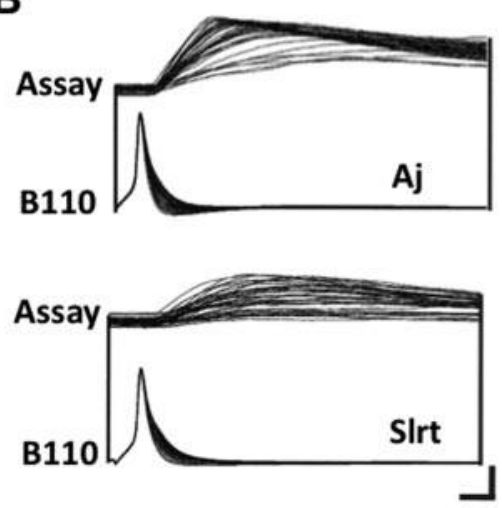

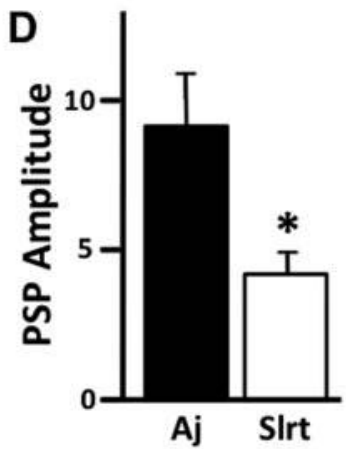

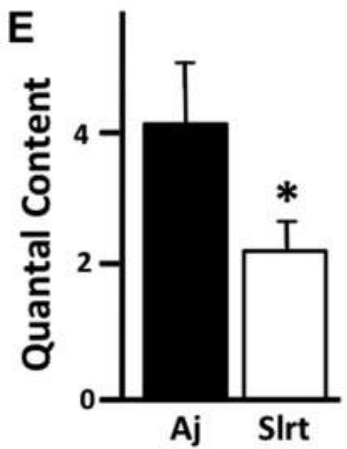

Figure 8. Synaptic properties of B110 following Aj and Slrt muscle contact. (A) Assay-cell recordings made from a neuron B110 contacting both $\mathrm{Aj}$ (top trace) and Slrt muscle fibers (bottom trace) illustrate the frequency and amplitude of mPSPs in representative preparations. Vertical scale bar equals $5 \mathrm{mV}$. Horizontal scale bar equals $500 \mathrm{~ms}$. (B) These traces illustrate 25 superimposed recordings from assay cells in contact with the neurites of a B110 with Aj muscle contact (top trace) and Slrt contact (bottom trace). Large EJPs were recorded in assay cells associated with the Aj-contacted neurites. Vertical scale bar equals $2 \mathrm{mV}$. Horizontal scale bar equals $20 \mathrm{~ms}$. (C) Average mPSP amplitudes in B110-Aj $(n=18$ preparations $)$ and B110-Slrt $(n=$ 19 preparations) experimental groups were not significantly different $(P=0.25)$. (D) PSP amplitudes, on the other hand, were different between experimental groups, with PSPs larger in assays of Aj-contacted neurons (*, $P<0.01$ ). (E) Quantal content was also different between experimental groups and was greater in Aj-contacted neuronal synapses $(*, P<0.04)$.

two neurons varies from vigorous muscle contraction to tonus during coordination of feeding muscle movements. Although these neurons function during different phases of the Helisoma feeding pattern, B19 and B110 innervate virtually identical sets of buccal muscles. Still, the percentage of individual fibers within a specific muscle group innervated by these motoneurons varies considerably. For example, B19-evoked EJPs were detected in more than $80 \%$ of Slrt muscle fibers of the ipsilateral musculature. In contrast, B110-evoked EJPs were detected in less than $40 \%$ of Slrt fibers of the contralateral musculature (Fig. 3). Furthermore, the efficacy of evoked-EJPs and the strength of the muscle contractions they activate are different at specific buccal neuron-muscle connections. Buccal neuromuscular synapses in Aplysia also have variable strength, and in some cases, no obvious contractions are elicited even though innervation is confirmed (Jordan et al., 1993). Thus, differential innervation patterns, combined with differential syn- aptic efficacy and potential for neuromodulatory plasticity, provides for a dynamic repertoire of buccal feeding apparatus movements generated from small sets of motoneuronal and neuromodulatory inputs.

The Slrt muscle groups are the most massive muscles of the Helisoma buccal apparatus (Kater, 1974). During contraction, radular muscles push the odontophore forward and provide a solid structure against which other muscles can exert the tension necessary for rasping movements of the radula (Yoshida and Kobayashi, 1991; Elliott and Susswein, 2002; Wentzell et al., 2009). The Aj is a large sheet-like muscle that extends anteriorly and dorsally over the buccal mass and functions to return the odontophore to its resting condition. One interesting point is the extensive contralateral connectivity of these neurons, demonstrating that motor outputs are coordinated on right and left sides by significant direct innervation, even though many buccal motor neurons are both ipsi- and contralaterally coupled by electrical net- 
works. It has been suggested that the redundancy in Helisoma bilateral coordination of motor outputs is due to both electrical coupling and bilateral axon projections, thus assuring synchronous bilateral function (Bahls et al., 1980).

The regeneration of buccal neurons often involves the formation of transient and novel electrical connections (Bulloch and Kater, 1981; Hadley et al., 1982) and the transient increase in coupling of extant electrical synapses between buccal neurons (Murphy et al., 1983). Similar forms of transient electrical coupling occur in the developing mammalian cortex (Kandler and Katz, 1998a, b) and during regeneration in the mammalian spinal cord after peripheral nerve injury (Chang and Balice-Gordon, 2000; Chang et al., 2000). Although these transient connections are thought to regulate neural network formation or reformation, their specific functions remain unknown. Nonetheless, transient electrical synaptogenesis alters the subsequent formation of chemical synapses (Szabo et al., 2004; Neunuebel and Zoran, 2005) and influences the outcome of neural network regeneration (Szabo and Zoran, 2007). Several days after axotomy, an electrical connection has formed between B19 and B110, which gradually diminishes in coupling strength and is eliminated by 5-7 days after nerve crush (Fig. 4). Electrical synchrony imposed by this transient electrical coupling might influence the synchrony of process outgrowth, the survival of damaged neurons, or activity-dependent mechanisms of synapse formation at central synapses. Neurite outgrowth in Helisoma buccal neurons is required for the formation of electrical connections between those neurons (Hadley and Kater, 1983; Hadley et al., 1983, 1985). Since growth-cone motility, filopodial function, and axon elongation of Helisoma motoneurons are calcium-dependent processes (Mattson and Kater, 1987; Rehder and Kater, 1992; Davenport and Kater, 1992) and process outgrowth is modulated by electrical activity of these neurons (Cohan and Kater, 1986; Cohan et al., 1987), it is likely that electrical activity in the population of electrically coupled neurons regulates their neurite outgrowth during regeneration. Thus, we suggest that this first stage of motoneuronal regeneration, characterized by axonal guidance to peripheral tissues and transient electrical coupling of buccal neurons, might require a special state of activity coordination among damaged neurons to promote the synchronous elongation of axons and the speedy arrival of growth cones at potential peripheral targets (Fig. 9A).

As the transient electrical communication between buccal neurons wanes, a unidirectional chemical connection is formed between neurons B110 to B19. Transient electrical coupling via gap junctions during development and regeneration may aid in the subsequent construction or maturation of chemical synapses (Montoro and Yuste, 2004; Kandler and Thiels, 2005; Marin-Burgin et al., 2006). This switch in electrical to chemical signaling is seen in both

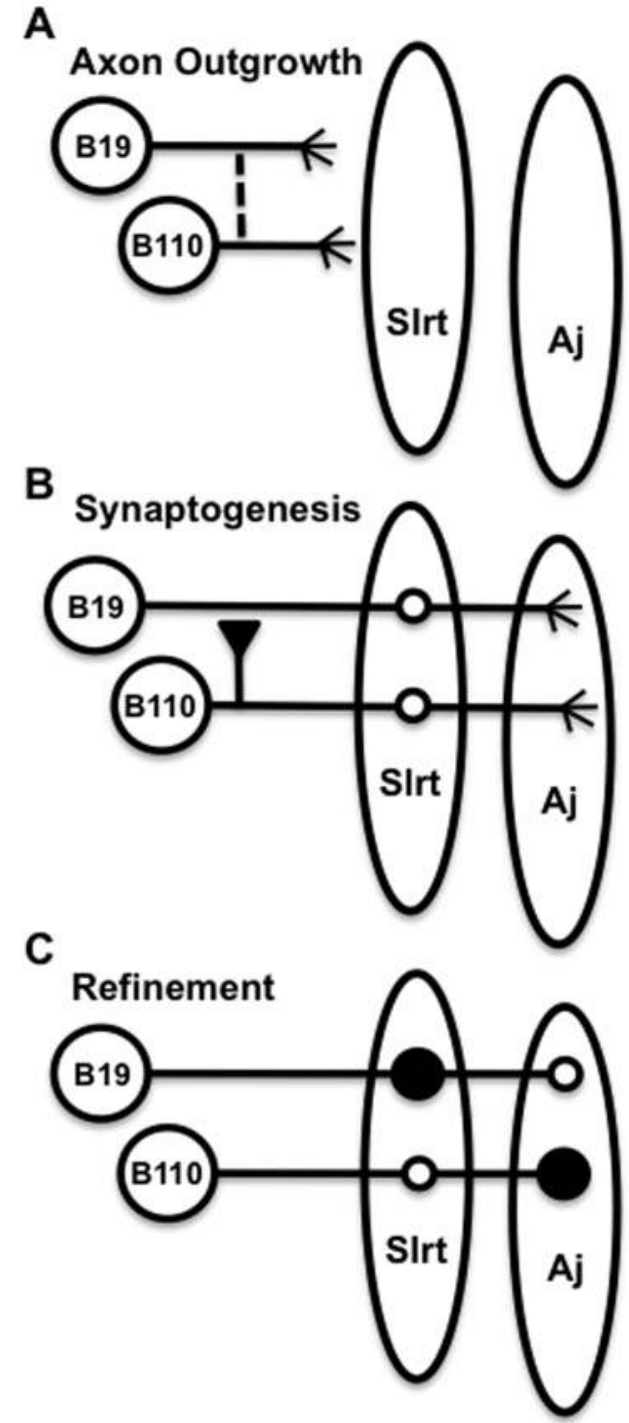

Figure 9. Model of buccal neuron regeneration in Helisoma. Three stages of regeneration involve different cellular mechanisms as Helisoma buccal motoneurons reestablish neuromuscular synapses. (A) During the stage of axonal outgrowth, transient electrical synapses form between neurons B19 and B110 (indicated by dashed line). Electrical coupling might facilitate axonal outgrowth to peripheral targets by synchronizing electrical activity that regulates growth-cone motility and axon elongation. (B) In the second stage of regeneration, chemical synaptogenesis occurs, with the formation of both an inhibitory connection between B110 and B19 (indicated by closed triange) and excitatory connections between motor neurons and muscle fibers (small open circles). Central inhibitory connections might facilitate synaptic competition in the periphery, which together with specific target recognition govern motoneuronal synaptogenesis. (C) The third and final stage involves the refinement and maturation of synapses, where neuromuscular-specific synaptic efficacies are established, perhaps through mechanisms of retrograde signaling and synaptic homeostasis. B19 forms stronger connections (indicated by large closed circles) with Slrt fibers, while B110 forms stronger synapses with Aj fibers.

mammalian (Arumugam et al., 2005) and molluscan nervous systems (Szabo et al., 2004) and involves an inverse 
relationship between chemical and electrical communication, a homeostatic regulation of synapse formation early in network formation or regeneration. The chemical connection formed between B110 and B19 is cholinergic and inhibitory (Szabo et al., 2004). Thus, when B110 is active, B19's activity might be suppressed by this inhibitory connection, but only after 5-7 days of regeneration (Fig. 4). Some Helisoma buccal neurons, such as B19, are constrained in their ability to regenerate synaptic connections, and the formation of a synapse on one target contact does not alter that neuron's synaptic function at another target (Poyer and Zoran, 1996). This differential regulation of synaptic efficacy requires recognition of an appropriate postsynaptic target (Zoran and Poyer, 1996). Therefore, the fact that neuron B19 does not also form a cholinergic chemical synapse onto B110 during regeneration is not surprising. Since B110 is known to be more promiscuous in its synapse formation, a strategy of synaptogenesis reported for other Helisoma buccal neurons (Haydon and Zoran, 1989), it possesses the capacity for rapid inhibitory synapse formation with other cholinoceptive neurons. The demonstration here that regeneration of identified buccal neurons in Helisoma involves dynamic plasticity of synaptic interactions is a further extension of our understanding that neural regeneration in invertebrate animals, particularly gastropod molluscs, incorporates many conserved and basic mechanisms that govern adult neuronal plasticity (Moffett, 2000). Furthermore, factors present in medium conditioned with damaged neural tissues influence the kinds of synapses that regenerate between molluscan neurons in cell culturefor example, chemical versus electrical or excitatory versus inhibitory (Hamakawa et al., 1999; Szabo et al., 2004).

We consider this period of transient inhibitory synapse formation, which coincides with neuromuscular synapse regeneration, to be the second stage of motoneuronal regeneration; that is, a stage of synaptogenesis following initial process outgrowth (Fig. 9B). The formation of a unidirectional inhibitory connection between B110 and B19 might provide an important central regulatory cue for peripheral neuromuscular regeneration. This inhibitory connection forms at precisely the time (6-8 days post-crush) when synaptic boutons and evoked EJPs appear in the peripheral musculature, and it remains strong and sustained until day 14 post-crush. Therefore, this transient inhibitory synapse would provide a mechanism for the inhibition of motoneuronal competitors during neuromuscular synapse regeneration. For example, inhibition of B19 would assure that its firing activity is asynchronous with that of B110, suggesting a role for activity-dependent synaptic competition during the regeneration of the Helisoma buccal motor system.

The present studies describe the time course for regeneration of B19 peripheral neuromuscular synapses. The first evidence of the presence of motoneuronal transmitter release onto buccal muscle is at 3-5 days after buccal nerve crush. Spontaneous mEJPs are first detected and then increase over this time period such that they are recorded in nearly $80 \%$ of muscle fibers tested. In contrast, B19-evoked EJPs are not detected at this time (Fig. 5). We call this period of regeneration Phase 1 (P1) neuromuscular synapse formation. By days 6-8 post-crush, weak B19-evoked EJPs are detected in Slrt muscle fibers, and spontaneous neuromuscular transmission is dramatically reduced (Fig. 5). We named this period Phase 2 (P2) neuromuscular synapse formation. Finally, starting at about days 9-11 and extending well into the second week after nerve injury, most muscle fibers that were innervated prior to injury become reinnervated, as demonstrated by the presence of B19evoked EJPs. In addition, synaptic efficacy is restored to pre-injury levels and spontaneous mEJPs are virtually absent. We call this period Phase 3 (P3) neuromuscular synapse formation. These phases of neuromuscular synapse formation are known to involve the elevation of resting calcium, voltage-activated calcium influx, and neurotransmitter release following contact of growth cones with muscle targets (Zoran et al., 1993; Funte and Haydon, 1993; Zoran and Poyer, 1996). Similar developmental and cascading events occur in vertebrate motoneurons after initial muscle contact (Dai and Peng, 1993) and at fly neuromuscular synapses (Littleton et al., 1993). Interestingly, actionpotential-evoked neurotransmitter release is significantly elevated following periods of sustained contact with appropriate Slrt muscle fibers, but not when mismatched with inappropriate muscle targets (Zoran and Poyer, 1996). This discrimination is likely based on target-specific recognition, as neurons are capable of distinguishing between synaptic targets through the recognition of cell surface cues in many animal systems (Johansen et al., 1989; Chiba et al., 1993; Merz and Drapeau, 1994; Bate and Broadie, 1995; Zhu et al., 1995). Therefore, neuromuscular synapse regeneration is an accurate mechanism, likely based on cell-cell signaling processes that regulate the emergence of spontaneous neuromuscular synaptic transmission (P1), the acquisition of intermittent evoked EJPs of weak efficacy (P2), and the formation of one-for-one, functional reinnervation (P3).

In our proposed model of buccal motoneuronal regeneration, the third and final stage is one of synaptic refinement, whereby homeostatic mechanisms of synaptic plasticity establish differential motoneuronal efficacy at muscles innervated by multiple neuronal inputs (Fig. 9C). Molluscan synapses regenerated in cell culture require ongoing and multiple modifications that affect functional maturation (Sun and Schacher, 1996; Conrad et al., 1999). Cell culture studies of isolated buccal neurons have established many of the cellular mechanisms governing the reformation of chemical and electrical synaptic connections (Haydon and Kater, 1988; Haydon and Zoran, 1989; Zoran and Poyer, 1996). Neuron B19 when stimulated in intact neuromuscu- 
lar preparations induces high-amplitude EJPs, associated with strong contractions of the Slrt muscle, and lower amplitude EJPs and weaker contractions of the Aj muscle (Fig. 6). The opposite synaptic relationships exist at B110 neuromuscular synapses. This differential synaptic physiology is recapitulated after neuronal synapse regeneration in vitro, where neuron B19 forms a stronger synaptic connection at Slrt rather than Aj contacts, and neuron B110 exhibits more efficacious neurotransmitter release at contact sites with $\mathrm{Aj}$ muscle fibers. These differences in motoneuronal presynaptic properties include a 2 -fold elevation in quantal content of neurotransmitter release at B110 sites of $\mathrm{Aj}$ muscle contact as compared to sites of Slrt contact (Fig. 8). These results suggest a role for retrograde influences of the muscle targets on presynaptic motoneuronal secretory mechanisms. It has long been understood that neuronal growth cones have the ability to recognize molecular cues on specific muscle targets with which they form synaptic connections (Chiba et al., 1995) and that these retrograde signals affect presynaptic transmitter release (Davis and Goodman 1998; Frank et al., 2006). Retrograde modulation of transmitter release properties occurs between isolated neurons and target cells in culture (Haydon and Zoran, 1994; Fernandez-de-Miguel and Drapeau, 1995). Our results on the maturation of buccal motoneuronal synapses suggest a role for retrograde influences of muscle targets on presynaptic motoneuronal secretory mechanisms, which ultimately shape the mature pattern of functional buccal muscle innervation. Therefore, we conclude that target-induced alterations in synaptic strength are an important final homeostatic stage in the regeneration of mature neuromuscular synapses.

In summary, a three-stage model is proposed to explain our observations of Helisoma buccal motoneuron regeneration. Transient electrical synaptogenesis might facilitate axonal outgrowth to peripheral targets during the first stage of recovery of function. In the second stage, differential motoneuronal synaptogenic capabilities, mechanisms of specific target recognition, and transient inhibitory chemical synpatogenesis act together to regulate the initial stages of neuromuscular synapse regeneration. The third and final stage of maturation involves the establishment of differential motoneuronal efficacies and employs mechanisms of both synaptic competition and synaptic homeostasis. The final maturation stage might be especially important in the regeneration of invertebrate neuromuscular systems, where muscle targets are innervated by multiple neuronal inputs. It remains to be seen how widespread the expression of transient electrical connections and transient inhibitory chemical connections is in regeneration of neural systems in general. It is clear that these transiently regenerated connections both affect future connectivity and are affected by existing and previous synaptic connections (Bulloch et al., 1984; Szabo and Zoran, 2007). However, our suggestion that one fundamental role of these transient connections is to regulate the regeneration of peripheral neuromuscular synapse requires further rigorous examination.

\section{Acknowledgments}

The authors thank J. Clay Gaspard for his research assistance with aspects of the electrophysiological analysis, and Jarret Richardson for his comments on the manuscript. This research was supported by National Science Foundation (IBN-9421372) and National Institutes of Health (P01 NS39546) grants to M. J. Z.

\section{Literature Cited}

Agata K., and Y. Umesono. 2008. Brain regeneration from pluripotent stem cells in planarian. Philos. Trans. R. Soc. Lond. B 363: 2071-2078.

Allison, P., and P. R. Benjamin. 1985. Anatomical studies of central regeneration of an identified molluscan interneuron. Proc. R. Soc. Lond. B 226: 135-157.

Arumugam, H., X. Liu, P. J. Colombo, R. A. Corriveau, and A. B. Belousov. 2005. NMDA receptors regulate developmental gap junction uncoupling via CREB signaling, Nat. Neurosci. 8: 1720-1726.

Bahls, F. H., S. B. Kater, and R. W. Joyner. 1980. Neuronal mechanisms for bilateral coordination of salivary gland activity in Helisoma. J. Neurobiol. 11: 365-739.

Bate, M., and K. Broadie. 1995. Wiring by fly: the neuromuscular system of the Drosophila embryo. Neuron 15: 513-525.

Bulloch, A. G., and S. B. Kater. 1981. Selection of a novel connection by adult molluscan neurons. Science 212: 79-81.

Bulloch, A. G., and S. B. Kater. 1982. Neurite outgrowth and selection of new electrical connections by adult Helisoma neurons. J. Neurophvsiol. 48: 569-583.

Bulloch, A. G., S. B. Kater, and H. R. Miller. 1984. Stability of new electrical connections between adult Helisoma neurons is influenced by preexisting neuronal interactions. J. Neurophvsiol. 52: 1094-1105.

Carrow, G. M., and I. B. Levitan. 1989. Selective formation and modulation of electrical synapses between cultured Aplysia neurons. J. Neurosci. 9: 3657-3664.

Chang, Q., and R. J. Balice-Gordon. 2000. Gap junctional communication among developing and injured motor neurons. Brain Res. Rev 32: $242-249$.

Chang, Q., A. Pereda, M. J. Pinter, and R. J. Balice-Gordon. 2000. Nerve injury induces gap junctional coupling among axotomized adult motor neurons. J. Neurosci. 20: 674-684.

Chiba, A., H. Hing, S. Cash, and H. Keshishian. 1993. Growth cone choices of Drosophila motoneurons in response to muscle fiber mismatch. J. Neurosci. 13: 714-732.

Chiba, A., P. Snow, H. Keshishian, and Y. Hotta. 1995. Fasciclin III as a synaptic target recognition molecule in Drosophila. Nature $\mathbf{3 7 4}$ $166-168$.

Cohan, C. S., and S. B. Kater. 1986. Suppression of neurite elongation and growth cone motility by electrical activity. Science 232: 1623 1640.

Cohan, C. S., J. A. Connor, and S. B. Kater. 1987. Electrically and chemically mediated increases in intracellular calcium in neuronal growth cones. J. Neurosci. 7: 3588-3599.

Conrad, P., F. Wu, and S. Schacher. 1999. Changes in functional glutamate receptors on a postsynaptic neuron accompany formation and maturation of an identified synapse. J. Neurobiol. 39: 237-248.

Dai, Z., and H. B. Peng. 1993. Elevation in presynaptic $\mathrm{Ca}^{2+}$ level accompanying initial nerve-muscle contact in tissue culture. Neuron 10: $827-837$. 
Davenport, R. W., and S. B. Kater. 1992. Local increases in intracellular calcium elicit local filopodial responses in Helisoma neuronal growth cones. Neuron 9: 405-416.

Davis, G. W., and C. S. Goodman. 1998. Synapse-specific control of synaptic efficacy at the terminals of a single neuron. Nature 392: 82-86.

Elliott, J. H., and A. J. Susswein. 2002. Comparative neuroethology of feeding control in mollusks. J. Exp. Biol. 205: 877-896.

Fernandez-de-Miguel, F., and P. Drapeau. 1995. Synapse formation and function: insights from identified leech neurons in culture. $\underline{\text { J. Neu- }}$ robiol. 27: 367-379.

Frank, C. A., M. J. Kennedy, C. P. Goold, K. W. Marek, and G. W. Davis. 2006. Mechanisms underlying the rapid induction and sustained expression of synaptic homeostasis. Neuron 52: 663-677.

French, K. A., and K. J. Muller. 1986. Regeneration of a distinctive set of axosomatic contacts in the leech central nervous system. $\underline{\text { J. Neurosci. }}$. 6: $318-324$.

Funte, L. R., and P. G. Haydon. 1993. Synaptic target contact enhances presynaptic calcium influx by activating cAMP-dependent protein kinase during synaptogenesis. Neuron 10: 1069-1078.

Hadley, R. D., and S. B. Kater. 1983. Competence to form electrical connections is restricted to growing neurites in the snail, Helisoma. J. Neurosci. 3: 924-932.

Hadley, R. D., R. G. Wong, S. B. Kater, D. L. Barker, and A. G. Bulloch. 1982. Formation of novel central and peripheral connections between molluscan central neurons in organ cultured ganglia. J. Neurobiol. 13: 217-230.

Hadley, R. D., S. B. Kater, and C. S. Cohan. 1983. Electrical synapse formation depends on interaction of mutually growing neurites. $\underline{\text { Science }}$ 221: $466-468$.

Hadley, R. D., D. A. Bodnar, and S. B. Kater. 1985. Formation of electrical synapses between isolated, cultured Helisoma neurons requires mutual neurite elongation. J. Neurosci. 5: 3145-3153.

Hamakawa, T., M. A. Woodin, M. C. Bjorgum, S. D. Painter, M. Takasaki, K. Lukowiak, G. T. Nagle, and N. I. Syed. 1999. Excitatory synaptogenesis between identified Lymnaea neurons requires extrinsic trophic factors and is mediated by receptor tyrosine kinases. J. Neurosci. 19: 9306-9312.

Haydon, P. G., and S. B. Kater. 1988. The differential regulation of formation of chemical and electrical connections in Helisoma. J. Neurobiol. 19: 636-655.

Haydon, P. G., and M. J. Zoran. 1989. Formation and modulation of chemical connections: evoked acetylcholine release from growth cones and neurites of specific identified neurons. Neuron 2: 1483-1490.

Haydon, P. G., and M. J. Zoran. 1991. Chemical synapses in cell culture. Pp. 57-71 in Cellular Neurobiology: A Practical Approach, J. Chad and H. Wheal, eds. Oxford University Press, New York.

Haydon, P. G., and M. J. Zoran. 1994. Retrograde regulation of presynaptic development during synaptogenesis. J. Neurobiol. 25: 694-706.

Haydon, P. G., D. P. McCobb, and S. B. Kater. 1987. The regulation of neurite outgrowth, growth cone motility, and electrical synaptogenesis by serotonin. J. Neurobiol. 18: 197-215.

Heyer, C. B., S. B. Kater, and U. L. Karllson. 1973. Neuromuscular systems in molluscs. Am. Zool. 13: 247-270.

Johansen, J., M. E. Halpern, and H. Keshishian. 1989. Axonal guidance and the development of muscle fiber-specific innervation in Drosophila embryos. J. Neurosci. 9: 4318-4332.

Jordan, R., K. P. Cohen, and M. D. Kirk. 1993. Control of intrinsic buccal muscles by motoneurons B11, B15, and B16 in Aplysia californica. J. Exp. Zool. 265: 496-506.

Kandler, K., and L. C. Katz. 1998a. Coordination of neuronal activity in developing visual cortex by gap junction-mediated biochemical communication. J. Neurosci. 18: 1419-1427.
Kandler, K., and L. C. Katz. 1998b. Relationship between dye coupling and spontaneous activity in developing ferret visual cortex. Dev. Neurosci. 20: $59-64$

Kandler, K., and E. Thiels. 2005. Flipping the switch from electrical to chemical communication. Nat. Neurosci. 8: 1633-1634.

Kaneko, C. R., M. Merickel, and S. B. Kater. 1978. Centrally programmed feeding in Helisoma: identification and characteristics of an electrically coupled premotor neuron network. Brain Res. 146: 1-21.

Kater, S. B. 1974. Feeding in Helisoma trivolvis: the morphological and physiological bases of a fixed action pattern. Am. Zool. 14: 1017-1036.

Littleton, J. T., H. J. Bellen, and M. S. Perin. 1993. Expression of synaptotagmin in Drosophila reveals transport and localization of synaptic vesicles to the synapse. Development 118: 1077-1088.

Liu, Y., and J. Nicholls. 1989. Steps in the development of chemical and electrical synapses by pairs of identified leech neurons in culture. Proc. R. Soc. Lond. B Biol. Sci. 236: 253-268.

Marin-Burgin, A., F. J. Eisenhart, W. B. Kristan, Jr., and K. A. French. 2006. Embryonic electrical connections appear to pre-figure a behavioral circuit in the leech CNS. J. Comp. Phvsiol. A Neuroethol. Sens. Neural. Behav. Phvsiol. 192: 123-133.

Martinez, V. G., G. J. Menger III, and M. J. Zoran. 2005. Regeneration and asexual reproduction share common molecular changes: upregulation of a neural glycoepitope during morphallaxis in Lumbriculus. Mech. Dev. 122: 721-732.

Matsuo, R., S. Kobayashi, J. Murakami, and E. Ito. 2010. Spontaneous recovery of the injured higher olfactory center in the terrestria slug Limax. PLoS One 5: e9054.

Mattson, M. P., and S. B. Kater. 1987. Calcium regulation of neurite elongation and growth cone motility. J. Neurosci. 7: 4034-4043.

McCobb, D. P., P. G. Haydon, and S. B. Kater. 1988. Dopamine and serotonin inhibition of neurite elongation of different identified neurons. J. Neurosci. Res. 19: 19-26.

Merz, D. C., and P. Drapeau. 1994. Segmental specificity of neuronal recognition during synapse formation between identified leech neurons. J. Neurosci. 14: 4125-4129.

Moffett, S. B. 1996. Nervous System Regeneration in the Invertebrates. Springer-Verlag, Berlin.

Moffett, S. B. 2000. Regeneration as an application of gastropod neural plasticity. Microsc. Res. Tech. 49: 579-588.

Montoro, R. J., and R. Yuste. 2004. Gap junctions in developing neocortex: a review. Brain Res. Brain Res. Rev. 47: 216-226.

Morgan, T. H. 1901. Regeneration. MacMillan, New York.

Murphy, A. D. 2001. The neuronal basis of feeding in the snail, Helisoma, with comparisons to selected gastropods. Prog. Neurobiol. 63: $383-408$.

Murphy, A. D., and S. B. Kater. 1978. Specific reinnervation of a target organ by a pair of identified molluscan neurons. Brain Res. 156: 322-328

Murphy, A. D., and S. B. Kater. 1980a. Differential discrimination of appropriate pathways by regenerating identified neurons in Helisoma. J. Comp. Neurol. 190: 395-403.

Murphy, A. D., and S. B. Kater. 1980b. Sprouting and functional regeneration of an identified neuron in Helisoma. Brain Res. 186 251-272.

Murphy, A. D., R. D. Hadley, and S. B. Kater. 1983. Axotomyinduced parallel increases in electrical and dye coupling between identified neurons of Helisoma. J. Neurosci. 3: 1422-1429.

Murphy, A. D., D. L. Barker, J. F. Loring, and S. B. Kater. 1985. Sprouting and functional regeneration of an identified serotonergic neuron following axotomy. J. Neurobiol. 16: 137-151.

Murrain, M., A. D. Murphy, L. R. Mills, and S. B. Kater. 1990. Neuron-specific modulation by serotonin of regenerative outgrowth and intracellular calcium within the CNS of Helisoma trivolvis. J. Neurobiol. 21: 611-618. 
Myohara, M., C. Yoshida-Noro, F. Kobari, and S. Tochitani. 1999. Fragmenting oligochaete Echytraeus japonensis: a new material for regeneration study. Dev. Growth Differ. 41: 549-555.

Neunuebel, J. P., and M. J. Zoran. 2005. Electrical synapse formation disrupts calcium-dependent exocytosis, but not vesicle mobilization. Svnapse 56: 154-165.

Nicholls, J. G., Y. Liu, B. W. Payton, and D. P. Kuffler. 1990. The specificity of synapse formation by identified leech neurones in culture. J. Exp. Biol. 153: 141-154.

Poyer, J. C., and M. J. Zoran. 1996. Activity-dependent induction of functional secretory properties at cultured neuromuscular synapses of Helisoma. J. Neurophysiol. 76: 2635-2643.

Price, C. J., and J. I. Goldberg. 1993. Serotonin activation of a cyclic AMP-dependent sodium current in an identified neuron from Helisoma trivolvis. J. Neurosci. 13: 4979-4987.

Purves, D., and J. W. Lichtman. 1985. Principles of Neural Development. Sinauer Associates, Sunderland, MA.

Ready, D. F., and J. Nicholls. 1979. Identified neurones isolated from leech CNS make selective connections in culture. Nature 281: 67-69.

Rehder, V., and S. B. Kater. 1992. Regulation of neuronal growth cone filopodia by intracellular calcium. J. Neurosci. 12: 3175-3186.

Ross, T. L., C. K. Govind, and M. D. Kirk. 1994. Neuromuscular regeneration by buccal motoneuron B15 after peripheral nerve crush in Aplysia californica. J. Neurophysiol. 72: 1897-1910.

Schacher, S., V. F. Castellucci, and E. R. Kandel. 1988. cAMP evokes long-term facilitation in Aplysia sensory neurons that requires new protein synthesis. Science 240: 1667-1669.

Snyder, K. A., and S. B. Moffett. 1990. Locomotion in the pulmonate snail Melampus-II. Recovery after pedal ganglion excision. Comp. Biochem. Physiol. A Physiol. 96: 407-414.

Sun, Z.-Y., and S. Schacher. 1996. Development of short-term heterosynaptic facilitation at Aplysia sensorimotor synapses in vitro is accompanied by changes in the functional expression of presynaptic serotonin receptors. J. Neurophysiol. 76: 2250-2261.

Syed, N. I., A. G. M. Bulloch, and K. Lukowiak. 1990. In vitro reconstruction of the respiratory central pattern generator of the mollusk Lymnaea. Science 250: 282-285.

Syed, N. I., A. G. M. Bulloch, and K. Lukowiak. 1992. The respiratory central pattern generator (CPG) of Lymnaea reconstructed in vitro. Acta Biol. Hung. 43: 409-419.

Syed, N. I., I. Roger, R. L. Ridgway, L. G. Bauce, K. Lukowiak, and A. G. Bulloch. 1993. Identification, characterisation and in vitro reconstruction of an interneuronal network of the snail Helisoma trivolvis. J. Exp. Biol. 174: 19-44.

Szabo, T. M., and M. J. Zoran. 2007. Transient electrical coupling regulates formation of neuronal networks. Brain Res. 1129: 63-71.

Szabo, T. M., D. S. Faber, and M. J. Zoran. 2004. Transient electrical coupling delays the onset of chemical neurotransmission at developing synapses. J. Neurosci. 24: 112-120.

Wentzell, M. M., C. Martínez-Rubio, M. W. Miller, and A. D. Murphy. 2009. Comparative neurobiology of feeding in the opisthobranch sea slug, Aplysia, and the pulmonate snail, Helisoma: evolutionary considerations. Brain Behav. Evol. 74: 219-230.

Yoshida, M., and M. Kobayashi. 1991. Neural control of the buccal muscle movement in the African giant snail Achatina fulica. J. Exp. Biol. 155: 415-433.

Zhu, H., F. Wu, and S. Schacher. 1995. Changes in expression and distribution of Aplysia cell adhesion molecules can influence synapse formation and elimination in vitro. J. Neurosci. 15: 4173-4183.

Zoran, M. J., and J. Poyer. 1996. Cellular mechanisms governing synapse formation: lessons from identified neurons in culture. Invertebr. Neurosci. 2: 1-8.

Zoran, M. J., and J. C. Poyer. 1999. Invertebrate neurons: Helisoma giant neurons. Pp. 643-650 in The Neuron in Tissue Culture, L. W. Haynes, ed. John Wiley, New York.

Zoran, M. J., P. G. Haydon, and P. J. Matthews. 1989. Aminergic and peptidergic modulation of motor function at an identified neuromuscular junction in Helisoma. J. Exp. Biol. 142: 225-243.

Zoran, M. J., R. T. Doyle, and P. G. Haydon. 1990. Target-dependent induction of secretory capabilities in an identified motoneuron during synaptogenesis. Dev. Biol. 138: 202-213.

Zoran, M. J., L. R. Funte, S. B. Kater, and P. G. Haydon. 1993. Neuron-muscle contact changes the presynaptic resting calcium setpoint. Dev. Biol. 158: 163-171.

Zoran, M. J., B. A. Metts, and J. C. Poyer. 1996. Specific muscle contacts induce increased transmitter release and neuritic arborization in motoneuronal cultures. Dev. Biol. 179: 212-222. 